\title{
Serine/Threonine-Protein Kinase NLK
}

National Cancer Institute

\section{Source}

National Cancer Institute. Serine/Threonine-Protein Kinase NLK. NCI Thesaurus. Code C90154.

Serine/threonine protein kinase NLK (527 aa, 58 kDa) is encoded by the human NLK gene. This protein is involved in the mediation of protein phosphorylation that affects cellular differentiation, transcription and signal transduction. 